\title{
RIMCIS
}

Hipatia Press

Instructions for authors, subscriptions and further details:

http://rimcis.hipatiapress.com

\section{De la Escuela Gueto a una Comunidad de Aprendizaje: Un Estudio de Caso sobre la Superación de la Pobreza a través de una Educación de Éxito}

Sandra Girbés-Peco ${ }^{1}$, Fernando Macías-Aranda ${ }^{1}$, Pilar ÁlvarezCifuentes $^{1}$

1) University of Barcelona, Spain

Date of publication: March $30^{\text {th }}, 2015$

Edition period: March 2015 - July 2015

To cite this article: Girbés, S., Macías, F., \& Álvarez, P. (2015). De la Escuela Gueto a una Comunidad de Aprendizaje: Un Estudio de Caso sobre la Superación de la Pobreza a través de una Educación de Éxito.

International and Multidisciplinary Journal of Social Sciences, 4(1), 88-116. doi : 10.17583/rimcis.2015.04

To link this article: http://dx.doi.org/10.17583/rimcis.2015.04

\section{PLEASE SCROLL DOWN FOR ARTICLE}

The terms and conditions of use are related to the Open Journal System and to Creative Commons Attribution License (CC-BY). 


\section{From a Ghetto School to a Learning Community: A Case Study on the Overcoming of Poverty through a Successful Education}

Sandra Girbés-Peco

University of Barcelona

Fernando Macías-Aranda

University of Barcelona
Pilar Álvarez-Cifuentes

University of Barcelona

\section{Abstract}

The increase of poverty in many European neighbourhoods in the last years has made public agendas and international research projects focus their efforts on identifying actions that effectively lead to stop the advancement of this social concern. In this context, the present article provides some of the contributions of the project from the Spanish National R\&D Programme "Successful Socio-educational Actions for overcoming poverty" (Valls, 20122014), regarding the combat against poverty and social exclusion through education. Particularly, this article focus on the results obtained in a case study located in one of the most disadvantaged areas of Europe, "La Milagrosa" neighbourhood (Albacete, Spain), and on the impact generated by the transformation of a school situated in this context into a Learning Community. The results of this study show that the Successful Educational Actions implemented there are enabling to overcome the educational exclusion that people living in this neighbourhood suffered. The transformation of the school into a Learning Community has brought new educational opportunities from early ages up to post-compulsory education, which is contributing to break the poverty circles that had become chronic in the neighbourhood.

Keywords: poverty, ghetto, Successful Educational Actions, Learning Communities 


\section{De la Escuela Gueto a una Comunidad de Aprendizaje: Un Estudio de Caso sobre la Superación de la Pobreza a través de una Educación de Éxito}

Sandra Girbés

Universidad de Barcelona
Pilar Álvarez-Cifuentes

Universidad de Barcelona

Fernando Macías-Aranda

Universidad de Barcelona

\section{Resumen}

El incremento de la pobreza en muchos y barrios europeos en los últimos años ha conducido a que las agendas políticas y las investigaciones internacionales concentren sus esfuerzos en identificar actuaciones efectivas que conduzcan a detener el avance de esta grave problemática. En este contexto, el presente artículo presenta algunas de las contribuciones del proyecto del Plan Nacional I+D “Actuaciones Socioeducativas de éxito para la superación de la pobreza" (Valls, 2012-2014) en relación a la lucha contra la pobreza a través de la educación. Concretamente, nos centramos en los resultados obtenidos durante el estudio de caso realizado en una de las áreas más desfavorecidas de Europa, el barrio de La Milagrosa (Albacete, España), y en el impacto generado por la transformación de la escuela situada en este contexto en una Comunidad de Aprendizaje. Los resultados de este estudio apuntan a que las actuaciones educativas de éxito aplicadas están posibilitando la superación de la exclusión educativa que sufrían los vecinos y vecinas. Transformar la escuela en una Comunidad de Aprendizaje ha supuesto generar nuevas oportunidades educativas desde edades tempranas hasta la educación postobligatoria, elemento que está contribuyendo a la ruptura de los círculos de pobreza que se habían cronificado en el barrio.

Palabras clave: pobreza, gueto, Actuaciones Educativas de Éxito, Comunidades de Aprendizaje 


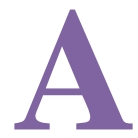

pesar de que Europa es considerada una de las zonas más prósperas del mundo, los datos obtenidos en los últimos años reflejan un aumento de la pobreza y el empeoramiento de las condiciones de vida de una parte importante de la ciudadanía. En este sentido, si prestamos atención a índices como AROPE ("At Risk Of Poverty and Exclusion") observamos que en 2013 el 24,5\% de la población (124,3 millones de personas) vivía en riesgo de pobreza y exclusión social (Eurostat, 2015). La situación de crisis financiera y económica experimentada en los últimos años está dificultando que se reviertan estas tendencias $\mathrm{y}$ ha generado consecuencias especialmente alarmantes en algunas regiones europeas. Por ejemplo, el índice AROPE muestra que en España el riesgo a sufrir situaciones de pobreza y exclusión social afecta a 12,8 millones de personas, lo que equivale a un $27,3 \%$ de la población (Eurostat, 2015). Según FOESSA (2014) la característica principal de la evolución de la desigualdad en el contexto español sigue siendo su rápido ascenso.

Ante esta situación, la investigación en el campo de las ciencias sociales se enfrenta al reto de ir más allá del diagnóstico de las situaciones de pobreza y exclusión, dando un paso adelante en la identificación de actuaciones efectivas que contribuyan a la formulación de políticas y medidas basadas en evidencias y orientadas a la resolución de los problemas sociales. Este imperativo ha evidenciado la necesidad de desarrollar mecanismos eficaces que evalúen el impacto de la investigación (Flecha, 2014-2018) en la mejora de las condiciones de vida de los colectivos tradicionalmente excluidos y que visibilicen aquellos estudios que están contribuyendo a la creación de nuevas realidades sociales de éxito (Aiello \& Joanpere, 2014).

Partiendo del giro que están experimentando las ciencias sociales, este artículo aporta algunos de los resultados derivados del proyecto del Plan Nacional I+D “Actuaciones socioeducativas de éxito para la superación de la pobreza” (Valls, 2012-2014). Esta investigación, desarrollada a partir de la colaboración de 19 investigadores e investigadoras de 11 universidades diferentes, tiene como finalidad identificar aquellas actuaciones que están aportando un impacto positivo en diferentes áreas sociales para la superación de la pobreza en barrios españoles con altos índices de pobreza y exclusión. 
Los objetivos del proyecto son (1) identificar actuaciones socioeducativas de éxito en la superación de la pobreza, es decir, aquellas estrategias que han aportado evidencias en relación a la superación de la pobreza y (2) aportar orientaciones en la elaboración de políticas públicas que contribuyan a la superación de la pobreza en España.

En este artículo aportamos los resultados obtenidos en relación a la aplicación de actuaciones educativas que han demostrado tener un impacto positivo en la superación de las trayectorias que conducen a la pobreza. Para ello, nos centramos en el estudio de caso de la escuela La Paz y en las evidencias obtenidas en este contexto sobre cómo la transformación de un centro educativo en una Comunidad de Aprendizaje está contribuyendo a activar estrategias educativas efectivas para toda la comunidad. En primer lugar, introducimos brevemente el estado de la cuestión y el marco teórico en el que se ha fundamentado esta investigación. En segundo lugar hacemos referencia a la metodología comunicativa, perspectiva utilizada en el desarrollo del estudio. En tercer lugar, presentamos los principales resultados extraídos del estudio de caso seleccionado. Por último, en la discusión se destaca cómo el caso de La Paz aporta orientaciones sobre cómo transformar escuelas de fracaso y exclusión en espacios generadores de oportunidades educativas y sociales para toda la comunidad.

\section{Marco Teórico}

Existe un amplio número de investigaciones científicas que han abordado el aumento de las desigualdades sociales (Sen, 2000; Nolan \& Whelan, 2010; Green \& Hulme, 2005). Entre éstas, se observa un consenso en el abandono de aquellas concepciones unidimensionales o reduccionistas de la pobreza identificadas en el pasado, a favor de aproximaciones multidimensionales que sitúan esta problemática como una situación forzada que no responde a la libre elección de los sujetos. De este modo, autores como Sen (2000) apuntan que la pobreza hace referencia a los recursos de los que disponen los sujetos no sólo en términos monetarios o de percepción de ingresos, sino también en función de la capacidad de las personas para acceder a una educación de calidad, mantenerse sanas, insertarse en el mercado laboral, sentirse seguras, tener un nivel de vida adecuado o poder participar en 
espacios de la vida pública. Si las situaciones de pobreza y exclusión son graves, lo es todavía más la irrupción de factores que están contribuyendo a su concentración en el espacio y su cronificación (Wilson, 2010), como por ejemplo las dinámicas macroeconómicas que han conducido al incremento dramático del desempleo. Estos fenómenos están afectando especialmente a los colectivos vulnerables entre los que destacan personas pertenecientes a minorías culturales o con bajos niveles formativos (Wilson, 2003). Además de los factores estructurales, existen investigaciones que se han centrado en los factores culturales asociados a la pobreza y la exclusión (Wilson, 2010). Estos se traducen en prácticas, interacciones y procesos de socialización que condicionan los planteamientos vitales y las decisiones de las personas que viven en barrios desfavorecidos y que se relaciona con la estereotipación, la estigmatización y las bajas expectativas. Diversos estudios se han centrado en cómo la combinación de factores adversos como los descritos generan un impacto negativo en el bienestar físico y emocional (Cohen, Farley \& Mason, 2003) de los individuos pero también en términos de cohesión social. En este sentio, actualmente contamos con multitud de casos (Espada \& Marimon, 2003; Gereluk \& Race, 2007) que ejemplifican cómo la concentración de minorías culturales (Orfield \& Eaton, 1996) en barrios con bajos niveles económicos y altas tasas de desempleo conduce al surgimiento de problemas de convivencia, violencia y disturbios.

La complejidad y el carácter dinámico de la pobreza (Alkire \& Santos, 2011; Klugman, Rodríguez, \& Choi, 2011) apunta a la necesidad de diseñar instrumentos efectivos que contribuyan a derribar aquellas barreras que han dificultado la superación de la pobreza y que han conducido a que muchas inversiones destinadas a estos fines hayan sido incapaces de mejorar las condiciones de vida de los colectivos vulnerables. En este sentido, diversos autores han identificado aquellos elementos que sí conducen al éxito de las intervenciones como por ejemplo elaborar políticas y acciones contra la pobreza basadas en evidencias científicas (Ríos, 2013) y contar con las voces de las personas implicadas en las problemáticas sociales (Valls \& Padrós, 2011). 


\section{La Lucha contra la Pobreza a través de la Educación}

Tanto investigaciones científicas (Santa Cruz, Siles \& Vrecer, 2011; Wolbers, 2003) como recomendaciones internacionales (European Commission, 2010; European Parliament, 2008; European Union Agency for Fundamentals Rights, 2009) sobre pobreza y exclusión social enfatizan dos circunstancias que incrementan la vulnerabilidad ante la pobreza: la falta de formación académica y la precariedad e inactividad laboral. La correlación entre el nivel de estudios alcanzados y las tasas de actividad laboral ya ha sido comprobada empíricamente (EUROSTAT, 2014; INE 2015) y, por ejemplo, en el contexto español se identifica que la tasa de actividad pasa de $9,12 \%$ entre las personas con estudios primarios no finalizados a un $81,97 \%$ entre las personas con titulación superior (INE, 2015).

Actualmente, existe cierto consenso a nivel internacional en relación a cómo las escuelas pueden marcar una diferencia en las trayectorias vitales de las personas, y en consecuencia también en el conjunto de la sociedad (DeLuca \& Rosenblatt, 2010; Giroux \& Flecha, 1992; Rose \& Dyer, 2008), superando los enfoques reproduccionistas que contemplaban la escuela como un espacio reproductor de las desigualdades sociales (Baudelot \& Establet, 1976; Jencks et al., 1979). En este sentido, en la última década, la literatura científica ha hecho especial énfasis en las actuaciones educativas que previenen y revierten las dinámicas de exclusión desde la primera infancia (Aikens \& Barbarin, 2008; Davidse, Dejong, Bus \& Lowenstein, 2011; Fantuzzo et al., 2004; Phillips \& Lowenstein, 2011; Stein et al., 2013). Una de las estrategias educativas que mayor impacto ha generado en la superación de la pobreza es que los niños y las niñas menores de seis años tengan la oportunidad de acceder a recursos y programas de desarrollo cognitivo y socioemocional. Además, el establecimiento de un currículo de máximos, especialmente en las áreas instrumentales (Maulucci, 2010; Hammond, 2001) o la extensión del tiempo de aprendizaje (Arnold \& Doctoroff, 2003; Mahoney, Lord \& Carryl, 2005; Taylor, Power \& Rees, 2010) son factores esenciales para el éxito académico y el acceso a la 
educación post-obligatoria (Baum \& Flores, 2011) de las personas que viven en contextos empobrecidos.

La literatura científica existente subraya la participación de las familias y de las personas de la comunidad en los centros educativos como una de las actuaciones que contribuyen al éxito educativo y al desarrollo del alumnado (Epstein, 1991, 1992; Henderson \& Mapp, 2002; Hidalgo, Epstein \& Siu, 2002; Huat See \& Gorard, 2013). A pesar del potencial que reside en el vínculo entre comunidad y escuela, ciertos estudios han apuntado a que no todos los tipos de participación generan el mismo impacto (Jeynes, 2012) y que incluso algunos intentos de promover la participación comunitaria han producido resultados indeseados como desconfianza entre la comunidad educativa o enfrentamientos entre los diferentes agentes de la comunidad educativa (Fine, 1993). En este sentido, un número limitado de estudios (Apple \& Beane, 2007; Delgado-Gaitan, 2004) se ha centrado en la identificación de aquellas formas democráticas y efectivas de participación en contextos desfavorecidos, entre las que destaca el proyecto de investigación del VI Programa Marco INCLUD-ED (Flecha, 2006-2011). Este proyecto ha identificado, entre otros, estrategias que conducen a desarrollar el potencial de la comunidad en las escuelas incluso en los contextos más desfavorecidos y contando con la participación de los colectivos tradicionalmente excluidos (Carreón, Drake \& Barton, 2005). Entre estas estrategias, la participación educativa y decisiva han sido destacadas como aquellas que generan mejores resultados en el incremento del aprendizaje del alumnado y en la mejora de la cohesión social (Díez, Gatt \& Racionero, 2011). En primer lugar, en relación a la participación educativa, este proyecto ha aportado modalidades de formación de familiares y de la comunidad basadas en las necesidades y demandas de las personas participantes. Además, la inclusión de los miembros de la comunidad en las aulas (Elboj \& Niemelä, 2010; Valls \& Kyriakides, 2013) y otros espacios educativos ha demostrado aportar beneficios tanto curriculares como extracurriculares. En segundo lugar, el concepto de participación decisiva aportado por INCLUD-ED implica la inclusión de las voces de las personas de la comunidad en el centro educativo. De este modo, basándose en el principio de la inteligencia cultural (Flecha, 2000), las familias y otros 
miembros de la comunidad participan activamente en los procesos de toma de decisiones sobre aspectos relevantes de la vida escolar.

La revisión bibliográfica permite identificar que estas formas de participación, así como algunas de las estrategias descritas, están demostrando sus buenos resultados en escuelas reales como por ejemplo en las Comunidades de Aprendizaje (Flecha \& Soler, 2013; Gatt, Ojala \& Soler, 2011): centros educativos en los que se aplican las actuaciones educativas de éxito abaladas por la comunidad científica internacional e implicando a toda la comunidad. En 2015, existen más de 190 centros educativos en España (en los niveles de educación infantil, primaria, secundaria y de personas adultas) y más de 150 escuelas en América Latina que se han transformado en Comunidad de Aprendizaje. Los resultados positivos aportados se han evidenciado en escuelas situadas en diferentes contextos geográficos y socioeconómicos. A través del estudio de caso de la escuela La Paz, en este artículo ampliamos el conocimiento existente en relación al impacto que puede generar la transformación de una escuela en una Comunidad de Aprendizaje, incluso en los contextos educativos y sociales más depauperados.

\section{Metodología}

Esta investigación se ha llevado a cabo siguiendo los principios de la Metodología Comunicativa (MC) (Flecha \& Soler, 2014; Gómez, Puigvert, \& Flecha, 2011), la cual permite identificar aquellos elementos que contribuyen a perpetuar la desigualdad y la exclusión social así como aquéllos que contribuyen a su superación. En la MC el conocimiento surge a partir de la interacción entre las evidencias científicas aportadas por los y las investigadores y las personas de la comunidad, quienes desde su mundo de la vida (Habermas, 1984) han realizado aportaciones fundamentales para la interpretación de la realidad de su barrio. Este diálogo igualitario (Puigvert, Christou \& Holdford, 2012) ha permitido realizar un análisis intersubjetivo de la realidad que no únicamente ha aumentado la representatividad de las investigaciones, sino también la calidad de los resultados alcanzados. Además, la MC se caracteriza por la participación de los sujetos beneficiarios en todas las fases de la investigación. Por ejemplo, en el 
estudio de caso que hemos llevado a cabo, se ha contado con la participación de los vecinos y vecinas desde el diseño de los instrumentos de recogida de la información hasta la interpretación de los resultados finales. Dicha participación se ha materializado en forma de consejo asesor, donde estaban representados 8 vecinos y vecinas del barrio. Por nuestra parte, el equipo investigador ha aportado los conocimientos científicos desde disciplinas diversas en relación a la superación de la pobreza (sociología, trabajo social y educación, principalmente). De este modo, y a través de un diálogo intersubjetivo entre participantes y equipo investigador se han alcanzado acuerdos en relación al diseño de la investigación y a la interpretación de los resultados.

\section{Técnicas de Recogida y Análisis de la Información}

El proyecto del Plan Nacional I+D "Actuaciones Socioeducativas de éxito para la superación de la pobreza" (Valls, 2012-2014) se ha llevado a cabo a través de cuatro fases de trabajo. Concretamente, en este artículo se aportan algunos de los resultados obtenidos en la segunda fase del proyecto, en la que se ha realizado un estudio de caso en profundidad del barrio La Milagrosa (Albacete, España) y del centro educativo situado en este contexto, la escuela La Paz. Para elaborar dicho estudio de caso, en un primer término se realizó un análisis documental de datos secundarios con el fin de contextualizar y conocer en profundidad el barrio y obtener evidencias de su transformación, así como de las mejoras que se están consiguiendo. Este análisis documental, procedente de estadísticas, informes y memorias, servicios sociales, entidades, etc., nos ha permitido recoger evidencias de la transformación de la escuela y en su contexto.

Posteriormente a este análisis, se llevó a cabo el trabajo de campo cualitativo, en el que participaron diferentes personas del barrio (Tabla 1): jóvenes de entre 14 y 18 años, vecinos/as y familiares adultos, y profesionales y agentes implicados en la transformación del barrio (desde profesionales de la educación o del tercer sector, hasta responsables políticos). Esta fase nos permitió identificar un conjunto de actuaciones socioeducativas que están contribuyendo a la superación de la pobreza en el barrio de La Milagrosa. 
Tabla 1

Técnicas de recogida de la información aplicadas y perfiles de las personas participantes

\begin{tabular}{|c|c|c|}
\hline Técnicas & $\mathbf{N}^{\mathbf{0}}$ & Perfil \\
\hline $\begin{array}{l}\text { Entrevistas en profundidad } \\
\text { de orientación comunicativa }\end{array}$ & 4 & $\begin{array}{l}2 \text { responsables políticos } \\
2 \text { responsables de Servicios Sociales }\end{array}$ \\
\hline $\begin{array}{l}\text { Relatos de vida } \\
\text { comunicativos }\end{array}$ & 18 & $\begin{array}{l}8 \text { con jóvenes de } 14 \text { a } 18 \text { años } \\
10 \text { con familiares }\end{array}$ \\
\hline $\begin{array}{l}\text { Grupos de discusión } \\
\text { comunicativos }\end{array}$ & 5 & $\begin{array}{l}2 \text { con vecinos/as } \\
-1 \text { con jóvenes } \\
-1 \text { con personas adultas del barrio (con hijos/as) } \\
\text { - } 2 \text { con profesionales } \\
-1 \text { con profesorado } \\
\text { - } 1 \text { con profesionales de la intervención social } \\
\text { - } 1 \text { con personas vinculadas a entidades sociales } \\
\text { (participantes y/o voluntarios/as) }\end{array}$ \\
\hline TOTAL & 27 & \\
\hline
\end{tabular}

En total, se implementaron 27 técnicas de recogida de la información: 4 entrevistas en profundidad con orientación comunicativa; 18 relatos de vida comunicativos y 5 grupos de discusión comunicativos.

\section{Análisis de la Información Recogida}

Para el análisis de la información, realizado con la ayuda del programa informático de análisis cualitativo de datos Atlas-ti, se han tenido en cuenta dos dimensiones: la exclusora y la transformadora. La identificación de componentes exclusores y transformadores es propia de la MC, en tanto que pretende no sólo describir e interpretar la realidad, sino también aportar conocimiento que permita mejorar las condiciones de vida estudiadas. Para la dimensión exclusora se han identificado aquellas acciones e iniciativas llevadas a cabo en el barrio y que no han contribuido a la superación de la pobreza. En su lugar, para la dimensión transformadora, se han considerado aquellas actuaciones que sí están contribuyendo a la superación de la pobreza y de la exclusión social en el barrio.

Este análisis, además de las dimensiones exclusoras y transformadoras, se llevó a cabo teniendo en cuenta las cuatro áreas cruciales para el estudio de 
98 Girbés-Peco et al. - De Escuela Gueto a Comunidad de Aprendizaje

la pobreza identificadas ya en la primera fase del proyecto: educación, salud, empleo y vivienda.

Tabla 2

Cuadro de análisis de la información

\begin{tabular}{lllll}
\hline & Educación & Empleo & Salud & Vivienda \\
\hline Dimensión Exclusora & & & \\
$\begin{array}{l}\text { Acciones e iniciativas que } \\
\text { NO han contribuido a la } \\
\text { superación de la pobreza. }\end{array}$ & Código 1 & Código 2 & Código 3 & Código 4 \\
\hline $\begin{array}{l}\text { Dimensión } \\
\text { Transformadora }\end{array}$ & & & \\
$\begin{array}{l}\text { Acciones e iniciativas que } \\
\text { Sí han contribuido a la } \\
\text { superación de la pobreza. }\end{array}$ & Código 5 & Código 6 & Código 7 & Código 8 \\
\hline
\end{tabular}

Después de analizar todos los datos recogidos, a continuación se presentan algunos de los principales resultados que evidencian el impacto de las actuaciones educativas de éxito que configuran las Comunidades de Aprendizaje en relación a la superación de las desigualdades educativas y sociales a las que se enfrentan los vecinos y vecinas del barrio La Milagrosa.

\section{Resultados}

\section{Introducción al Estudio de Caso: El Barrio de La Milagrosa}

El barrio de La Milagrosa se encuentra situado en el sureste de Albacete (España), en la periferia de la ciudad. Fue creado durante los años 70 bajo una estrategia de realojo para personas que vivían en un asentamiento chabolista y personas de clase obrera. Con el tiempo, el barrio fue siendo ocupado por personas cada vez más vulnerables, lo que supuso un aumento de la pobreza y la exclusión, hasta convertirse en uno de los barrios gueto 
más pobre del Sur de Europa. Esta exclusión derivó en graves problemas de seguridad y en el aumento de la conflictividad (EAPN-CLM, 2010).

La población total del barrio de La Milagrosa es de 3.158 personas, lo que representa el 1,8\% de la población total de Albacete (INE, 2013). Es una población joven, ya que el $34 \%$ son personas menores de 15 años y el $62 \%$ personas de entre 15 y 65 años, en la que predominan los hombres $(51,11 \%)$ sobre las mujeres $(48,89 \%)$. Estos datos reflejan una esperanza de vida más corta y una vulnerabilidad mayor de las mujeres con respecto al resto de la población. A pesar de que en su mayor proporción la población del barrio es de etnia gitana o "merchera", en la actualidad el 11\% de la población es de origen extranjero (INE, 2013). Por lo que respecta al nivel socioeconómico del barrio, cabe destacar los altos índices de desempleo, la elevada tasa de analfabetismo y/o de personas sin estudios primarios, así como importantes problemas de salud derivados por la falta de recursos económicos o por las situaciones de drogodependencias (Brown, Gómez \& Munté, 2013).

Por lo que respecta a la oferta educativa que se ofrece en el barrio de La Milagrosa, encontramos el colegio público "La Paz", Comunidad de Aprendizaje desde el año 2006. Anteriormente, este centro era denominado escuela "San Juan", conocida por sus problemas de convivencia entre profesorado, alumnado y familias. Por ejemplo, esta escuela había aparecido varias veces en los medios de comunicación debido a que el profesorado era recibido con piedras por las familias, lo que les llevó a solicitar protección policial en varias ocasiones. Ante esta situación, y teniendo en cuenta los altos índices de fracaso escolar y absentismo de la escuela, la administración pública decidió cerrar el centro y reabrirlo como la escuela pública "La Paz" y bajo el proyecto educativo de Comunidades de Aprendizaje. Gracias a la apuesta por este proyecto fundamentado sobre actuaciones educativas de éxito y basado en la participación de la comunidad, como los grupos interactivos o las tertulias literarias dialógicas, actualmente, este centro es reconocido internacionalmente por la superación de las problemáticas

${ }^{1}$ Grupo de personas que han adquirido valores y costumbres similares a las del pueblo gitano, pero que sin embargo no comparten con éste su origen étnico. 
identificadas en las llamadas escuelas gueto (Díez-Palomar, Santos Pitanga \& Álvarez, 2013).

\section{El Impacto de las Actuaciones Educativas de Éxito}

Tal y como hemos presentado anteriormente, el acceso a recursos y programas de desarrollo cognitivo y socioemocional en la primera infancia es una de las estrategias detectadas en la literatura científica que previene la exclusión. En el estudio de caso analizado hemos identificado que la conversión de un centro educativo en Comunidad de Aprendizaje ha logrado transformar radicalmente el acceso a la escolarización formal de los niños y niñas de temprana edad. Como afirma una de las profesionales del ámbito social que interviene en el barrio, la transformación de la escuela ha permitido hacer frente a las dinámicas de abandono y fracaso educativo que se habían cronificado en el barrio:

El nivel de estudios sobre todo en la población más joven sí que creo que ha mejorado considerablemente. Se han abordado temas muy importantes que estaban pendientes de resolver como es el tema del absentismo o el de la escolarización. Creo que eso está prácticamente resuelto y la población más joven sí que creo que existe una evolución positiva importante. (Profesional del área social)

Los datos recogidos en el centro son elocuentes desde el punto de vista de la asistencia al centro (ver tabla 3). La escuela La Paz había sido un lugar marcado por altas tasas de absentismo y descenso de la matricula. Las personas del barrio tendían a enviar a sus hijos e hijas a escuelas de la periferia, o incluso mantenerlos fuera de la escuela, debido entre otros aspectos, a la fuerte conflictividad con el claustro de profesorado.

La tabla 3 sugiere que desde mediados de la década de 1990 hasta la mitad de la primera década del 2000, la escuela sufrió un proceso de abandono que llevó a la perdida de dos de cada tres estudiantes en su matrícula. El porcentaje de pérdida es tan elevado, que indica que no se debe a una situación coyuntural (i.e. descenso de la natalidad). Los datos demográficos del barrio donde está ubicada la escuela también desmienten 
una motivación coyuntural debida a algún cambio de tendencia poblacional. En cambio, tal y como se aprecia en los datos adjuntos, en 2005-2006 el centro reabre sus puertas como "Comunidad de Aprendizaje" y experimenta un cambio de tendencia: en seis años recupera más de la mitad del alumnado que se había "perdido" a lo largo de los diez años precedentes.

Tabla 3

Evolución de la matrícula del Colegio Público "La Paz"

\begin{tabular}{lcccc}
\hline Curso escolar & \multicolumn{4}{c}{ ALUMNADO MATRICULADO } \\
\hline & INF-PRIM & ESO & ADULTOS & TOTAL \\
\hline $1994-1995$ & 334 & - & - & 334 \\
$2005-2006$ & 100 & - & - & 100 \\
\hline El centro se convierte en COMUNIDAD DE APRENDIZAJE LA PAZ \\
\hline $2006-2007$ & 114 & - & - & 114 \\
$2007-2008$ & 129 & - & - & 129 \\
$2008-2009$ & 146 & 19 & - & 165 \\
$2009-2010$ & 145 & 35 & - & 180 \\
$2010-2011$ & 155 & 42 & - & 197 \\
$2011-2012$ & 164 & 58 & $42(1)$ & 264 \\
$2012-2013$ & 151 & 59 & $26(2)$ & 236 \\
\hline
\end{tabular}

1) Con dos aulas y dos maestras

2) Con un aula y una maestra

Fuente: Equipo de evaluación de CCBB CEIP La Paz, Albacete. (2012)

Se trata de un cambio cualitativamente crucial, que impregna no solo a la escuela, sino al resto del barrio, en aspectos tales como la "sensación de seguridad", "civismo" o "sentido de barrio". En la siguiente cita correspondiente a la madre de una alumna del centro podemos apreciar que esta tendencia a la transformación conduce a la reducción de situaciones propias de entornos de pobreza, como por ejemplo el tráfico y consumo de drogas o la conflictividad.

Ha mejorado mucho la convivencia. No hay mucha droga como había antes, tanta delincuencia, ha mejorado mucho, mucho. Antes era imposible ver a un hombre entrar en un colegio y sentarse con el hijo 
allí a ayudarle, ayudarle hacer las cuentas, ahora sí se ve. (Madre de una alumna del colegio "La Paz")

Uno de los datos extraídos del trabajo de campo que se relaciona con este elemento es que la escuela La Paz se ha convertido en un lugar de esperanza para las personas que viven en el barrio. De acuerdo con los datos recogidos, esto es así por el cambio de "imagen" que tiene el centro entre la población del barrio, entre otros motivos. Gracias a que en la Comunidad de Aprendizaje pueden entrar, participar y tomar decisiones, el centro se ha convertido en un lugar apreciado por las familias y por los miembros de la comunidad, que lo cuidan y lo tratan como si fuese su propia casa. Según un vecino del barrio, esto es así porque ahora "sus voces cuentan:"

Yo he notado mejoría en el colegio porque podemos entrar libremente al cole y antes no, a todos padres no dejaban entrar. Y luego el mediador, por ejemplo, discuten dos niños y los separan y todos han cambiado mucho. Para que veas la libertad, yo voy al cole y voy como Pedro por mi casa pero con el orden, y antes yo no conocía por dentro. (Padre de un alumno del colegio "La Paz")

A través de las entrevistas y de la revisión de documentos secundarios se ha identificado cómo la inclusión de las familias en la toma de decisiones del centro desembocó en una actuación clave en el curso 2008-2009: abrir una linea de matrícula para cursar la ESO en la propia escuela. Esta decisión es clave porque una de las razones de fracaso escolar detectadas en la investigación es que la mayor parte del alumnado abandonaba los estudios en la transición entre la educación primaria y la educación secundaria debido a la resistencia a salir "fuera del barrio." Esta problemática fue discutida en una de las asambleas organizadas en la escuela en la que las familias identificaron la inexistencia de un centro de secundaria dentro del barrio como uno de los elementos que perpetuaba la exclusión educativa de las personas de La Milagrosa. Además, en esta misma asamblea varias familias soñaron que sus hijos/as no tuvieran que salir del barrio para continuar sus estudios. Partiendo de las demandas de la comunidad, en 2008 la escuela de La Paz hizo realidad este sueño integrando la educación secundaria. De este modo, por primera vez en la historia de La Milagrosa, niños/as gitanos/as del 
barrio cursan la secundaria. Este hecho ilustra también el alcance de las actuaciones de éxito dado que, como sabemos a partir de trabajos de investigación previos, aumentar el nivel educativo contribuye a la superación de la pobreza (DeLuca \& Rosenblatt, 2010; Girourx \& Flecha, 1992; Rose \& Dyer, 2008).

Los datos extraídos de la investigación apuntan a que la escuela, reflejo del entorno social en el que se encuentra, mejora de manera radical sus condiciones a partir de su transformación en una Comunidad de Aprendizaje: es más segura, abandona enfoques compensatorios para centrarse en máximos aprendizajes y la convivencia mejora sensiblemente. En la siguiente cita extraída de un grupo de discusión con profesionales de distintas áreas sociales del barrio se refleja como jóvenes que antes abandonaban los estudios y entraban en "la vía de la prisión", ahora tienen la oportunidad de continuar sus estudios.

Nosotros llevamos diez años, doce años ya dando lo que era la antigua Garantía Social y ahora en los PCPI solo tenemos uno. Creo que con muy buenos resultados, el tema del acompañamiento de chicos y chicas que han terminado la secundaria, que todos los años se matriculan, chicos y chicas que estaban absolutamente excluidos del sistema educativo formal, de centros educativos, de institutos y tal. (Profesional del área social)

En las entrevistas realizadas se ha identificado cómo la escuela y el tipo de educación que ofrece ha transformado las expectativas de los y las jóvenes del barrio. Es importante enfatizar que no podemos afirmar que por el mero hecho de terminar una formación básica se puede indicar que la pobreza se ha superado; pero sí que ésa es una condición para lograrlo. Los diferentes perfiles de participantes entrevistados han coincidido en la percepción de que disponer de un centro de educación secundaria en el barrio realmente "abre posibilidades" para las personas que viven en él. En una sociedad en la que es necesario tener credenciales para poder pasar los procesos de selección que dan acceso al mercado de trabajo, como condición sine qua non para tener opciones de "salir del círculo de la pobreza", el hecho de obtener el nivel de educación básico es una garantía para poder optar a ese horizonte de posibilidades. Tal y como afirma el coordinador de 
una entidad de educación no formal en uno de los grupos de discusión realizados con profesionales del barrio, se han generado nuevas expectativas educativas y vitales para los y las jóvenes del barrio:

Que está muy bien acabar secundaria, está muy bien que [los y las jóvenes] tengan éxito, y es cierto, se ha conseguido un montón. Ya no hay absentismo, prácticamente es inexistente. Y casi todo el mundo, ellos, el objetivo de secundaria está en sus cabezas pero más allá es lo que se nos está olvidando, y eso está muy relacionado con el acceso al mercado laboral. (Coordinador de una entidad de educación no formal)

Para las familias, la escuela y la educación se han convertido en una apuesta clara como recurso para salir de la pobreza. Los datos recogidos a lo largo de tres años de trabajo de campo reafirman esta interpretación. A continuación, la cita de una joven gitana relata la importancia que ha adquirido la escuela en el barrio, hasta el punto que las familias, las mismas que antes "quitaban" a sus hijos de esa escuela, ahora se preocupan para que no pierdan ni una sola clase:

Mi padre me mata como no venga a estudiar. Porque mi padre me dice: "El día de mañana cuando no esté yo, a parte ¿qué haces en casa sin estudiar?, ¿qué vas a hacer de la vida?, ¿vas a estar siempre en casa?, ¿nunca te vas a buscar un trabajo? Estudia, tus conocimientos, el ser alguien en la vida no es ser una persona analfabeta. El que te pongan un papel en tu cole y que te digan: "pues no, esto es así y es así"." (Joven alumna del colegio La Paz)

Para finalizar, queremos aportar un dato que sugiere la necesidad de seguir profundizando en el estudio de caso abordado. Como hemos expuesto, en 2008 la participación educativa de la comunidad en la escuela La Paz había conducido a que familias, profesorado y responsables educativos apostaran por la implementación de la educación secundaria en el centro. En 2012, la importancia de la participación educativa se vuelve a manifestar a través de otra decisión clave: incorporar una escuela de personas adultas en el mismo centro educativo. Unos años después, en el curso 2012-2013, 26 personas adultas del barrio se matriculaban en clases de neolectores, 
alfabetización y educación secundaria. Además, la escuela ha generado una oferta de formación durante las tardes-noches que ha contribuido a que varias personas del barrio puedan obtener certificados de formación de carácter profesionalizador, abriéndoles las puertas del mercado de trabajo. La siguiente cita correspondiente a un vecino ilustra las dinámicas recorridas por diversas personas del barrio que han pasado de vivir en situaciones de pobreza a la inclusión educativa y social. Para estos vecinos y vecinas, la participación voluntaria en la escuela ha sido el primer paso para acceder a una formación reglada y, a su vez, ha sido la puerta de entrada al mercado laboral:

Yo he podido ser lo que soy ahora, que para mí no es mu... a ver, no es mucho pero para mí es mucho porque a través de esos cursos yo puedo trabajar en las escuelas de verano, los tres meses de verano siempre trabajo, y he trabajado tres años en el comedor escolar de aquí. (Vecino gitano del barrio)

Los datos recogidos testimonian este proceso de en el que se están asentando las bases para la superación de la pobreza y en el que la escuela es un protagonista claro.

\section{Discusión}

La literatura científica previa ha demostrado ampliamente que existe una correlación entre pobreza y educación (Giroux \& Flecha, 1992). Orfield y Lee (2005) afirman que la naturaleza de la segregación en educación es multidimensional. Partiendo de diversos estudios realizados, sobre todo, en Estados Unidos, ambos autores examinan la relación entre los contextos de privación (barrios gueto, regiones excluidas, etc.) y las altas tasas de fracaso escolar de las escuelas que se encuentran ubicadas en dichos contextos. La revisión de estudios previos que hizo Rothstein (2004) confirma que en entornos de exclusión existe una tendencia del profesorado de las escuelas a reducir las exigencias curriculares, adoptando medidas que conducen hacia una segregación del alumnado, cuyo resultado es el fracaso escolar.

Diversas de las dificultades con las que se encuentran las escuelas ubicadas en este tipo de barrios depauperados incluyen la alta volatilidad de la 
plantilla docente (que acostumbra a renovar más de la mitad de sus efectivos cada curso escolar), la conflictividad con el entorno, la aparente falta de motivación de buena parte del alumnado, poca participación de las familias, y decisiones estratégicas docentes orientadas a la segregación. Cuando se producen cualquiera de estas circunstancias, el resultado es que los niños y las niñas, en general, abandonan los estudios e incluso dejan de asistir al centro. El caso de la escuela estudiada en este artículo se corresponde con lo relatado hasta el curso 2005-2006. En ese momento, y a raíz del compromiso de adoptar actuaciones educativas de éxito, el círculo marcado por la pobreza y su conexión con una educación deficitaria, se rompe.

Los testimonios de las personas participantes en la investigación ilustran lo ya sugerido por los datos de la tabla 1. La escuela ha implementado actuaciones educativas de éxito tales como grupos interactivos o tertulias literarias dialógicas, entre otras, y ahora se está logrando que niños y niñas acaben sus estudios y tengan oportunidades de inserción en el mercado de trabajo. De acuerdo con Valls y Padrós (2011), la manera más efectiva de luchar contra la pobreza es incluir en la investigación y en las acciones para superar la pobreza las voces de las personas que la padecen. A través del caso estudiado vemos que la implicación de las familias y de otros miembros de la comunidad en el centro educativo, a través del proyecto de Comunidades de Aprendizaje, explica en gran medida los avances hacia el éxito académico. Esta participación se traduce en un cambio de la imagen que tiene la escuela en el barrio, además del incremento de oportunidades que aporta la educación, dado que las personas del barrio no solo ven cómo sus hijos e hijas mejoran sus niveles educativos y acaban la primaria (por primera vez en el barrio), sino que además tienen la opción de proseguir sus estudios de secundaria. Además, la escuela también se convierte en un espacio para la formación de personas adultas, elemento que abre también la posibilidad a que las personas adultas puedan mejorar sus competencias y certificaciones para acceder al mercado de trabajo (condición sine qua non para salir del círculo de la pobreza). Dado que el nivel educativo "protege" de la pobreza y de la exclusión, este tipo de iniciativas se revela como crucial en la lucha contra estos fenómenos.

La aplicación de actuaciones que han producido éxito en el pasado ha demostrado ser una excelente estrategia para lograr que escuelas ubicadas en 
zonas de pobreza consigan transformar su situación (Izumi, 2002; Flecha \& Soler, 2013). En el caso que describimos aquí, la clave está en la participación de toda la comunidad en un proyecto que tiene por objetivo atajar la situación de exclusión educativa y social de las personas del barrio y que para conseguirlo se sustenta sobre actuaciones avaladas científicamente.

\section{Referencias}

Aiello, E., \& Joanpere, M. (2014). Social Creation. A New Concept for Social Sciences and Humanities. RIMCIS-International and Multidisciplinary Journal of Social Sciences, 3(3), 297-313. doi: 10.4471/rimcis.2014.41

Aikens, N.L., \& Barbarin, O. (2008). Socioeconomic differences in Reading trajectories: The contribution of family neighborhood and school contexts. Journal of Educational Psychology, 100(2), 235-251. doi: 10.1037/0022-0663.100.2.235

Alkire, S., \& Santos, M. (2011). Acute Multidimensional Poverty: A New Index for Developing Countries. Berlin: German Development Economics Conference.

Apple, M. W., \& Beane, J. A. (2007). Democratic schools: Lessons for a powerful education (2nd ed.). Portsmouth, NH: Heinemann.

Arnold, D., \& Doctoroff, G. (2003). The early education of socioeconomically disadvantaged children. Annual Review of Psychology, 54, 517-45. doi:

10.1146/annurev.psych.54.111301.145442

Aubert, A., Flecha, A., García, C., Flecha, R., \& Racionero, S. (2008). Aprendizaje dialógico en la sociedad de la información. Barcelona: Hipatia.

Baudelot, C., \& Establet, R. (1976). La escuela capitalista en Francia (2a ed.). Madrid: Siglo XXI. 
108 Girbés-Peco et al. - De Escuela Gueto a Comunidad de Aprendizaje

Baum, S., \& Flores, S.M. (2011). Higher education and children in immigrant families. The Future of Children, 21(1), 171-193. doi:

\subsection{3/foc. 2011.0000}

Brown, M., Gómez, A., \& Munté, A. (2013). Procesos dialógicos de planificación de los servicios sociales: el proceso de cambio en los barrios de La Milagrosa y La Estrella (Albacete). Scripta Nova: Revista Electrónica de Geografía y Ciencias Sociales, 17 (277). Retrieved from: http://www.ub.edu/geocrit/sn/sn-427/sn-427-6.htm Caritas España (2013). VIII Informe del Observatorio de la Realidad Social. Madrid: Equipo de ESTUDIOS de Cáritas Española. Retrieved from: http://edit.um.es/exclusionsocial/files/2014/02/2013-

C\%C3\%A1ritas.pdf

Carreón, G., Drake, C., \& Barton, A. (2005). The importance of presence: Immigrant parents' school engagement experiences. American Educational Research Journal, 42(3), 465-498. doi:

10.3102/00028312042003465

Cohen, D., Farley, T., \& Mason, K. (2003). Why is poverty unhealthy?

Social and physical mediators. Social Science \& Medicine, 57(9), 1631-1641. doi: 10.1080/0141987032000132522

Comisión Europea (2010). Eurobarómetro especial sobre pobreza y exclusión social. Retrieved from:

http://ec.europa.eu/public_opinion/archives/ebs/ebs_355_en.pdf

Comisión Europea (2011). Eurobarómetro especial sobre empleo y política social. Retrieved from: http://ec.europa.eu/public_opinion/archives/ebs/ebs_377_fact_es_es.p df

Davidse, N., DeJong, M.T., Bus, A.G.S., C.J., \& Huijbregts, H.S. (2011). Cognitive and evironmental predictors of early literacy skills. Reading and Writing, 24 (4), 395-412. doi: 10.1007/s11145-010-9233-3

Delgado-Gaitan, C. (2004). Involving Latino families in schools: Raising student achievement through home-school partnerships. Thousand Oaks, CA: Corwin Press. 
DeLuca, S., \& Rosenblatt, P. (2010). Does moving to better neighborhoods lead to better schooling opportunities? Parental school choice in an experimental housing voucher program. The Teachers College Record, 112(5), 7-8. Retrieved from

http://www.grahamimac.com/housingandeducation/pdf/DelucaRosenb latt_2010.pdf

Díez, D., Gatt, S., \& Racionero, S. (2011). Placing immigrant and minority family and community members at the school's centre: The role of community participation. European Journal of Education, 46(2), 184196. doi: 10.1111/j.1465-3435.2011.01474.x

Díez-Palomar, J., Santos Pitanga, T., \& Álvarez Cifuentes, P. (2013). La Paz School. From a Ghetto to a Magnet School [Special issue]. International Review of Qualitative Research, 6(2), 198-209. doi: 10.1525/irqr.2013.6.2.198

EAPN-CLM. European Poverty Network/Castilla - La Mancha (2010). Exclusión Social en Castilla-La Mancha. Toledo: EAPN-CLM.

Elboj, C., \& Niemelä, R. (2010). Sub-communities of mutual learners in the classroom: The case of interactive groups. Revista De Psicodidáctica, 15(2). doi: 177-189. 10.1387/RevPsicodidact.810

Elboj, C., Pulido, M. A., \& Welikala, T. (2013). Las tecnologías de la información y la comunicación en la salida del aislamiento rural. El caso de Ariño. Scripta Nova. Revista Electrónica De Geografía Y Ciencias Sociales, XVII, 427 (2). Retrieved from: http://www.ub.edu/geocrit/sn/sn-427/sn-427-2.htm

Epstein, J. L. (1991). Effects on student achievement of teachers' practices of parent involvement. In S. B. Silvern (Ed.). Advances in Reading/Language Research: Literacy Through Family, Community, and School Interaction. Vol.5, (pp.261-276). US: Elsevier Science.

Epstein, J. L. (1992). School and family partnerships. In M. Aiken (Ed.). Encyclopedia of educational research. New York: Macmillan.

Espada, A., \& Marimon, S. (2003, 15 de mayo). Olvidar Ca n'Anglada. El País. 
110 Girbés-Peco et al. - De Escuela Gueto a Comunidad de Aprendizaje

European Commission (2010). Joint report on social protection and social inclusion. Luxembourg: Publications Office of the European Union.

European Commission (2011). Communication from the Commission to the

European Parliament, the Council, the European Economic and

Social Committe and the Committee of the regions. Tackling early

school leaving: A key contribution to the Europe 2020 Agenda.

Brussels: European Commission.

European Parliament (2008). Impact of cohesion policy on the integration of

vulnerable communities and groups. Brussels: European Union.

European Union Agency for Fundamentals Rights (2009). European Union

minorities and discrimination survey. Data in focus report the Roma.

Budapest: EU-MIDIS.

EUROSTAT (2014). People at risk of poverty or social exclusion. Retrieved from http://ec.europa.eu/eurostat/statistics-

explained/index.php/People_at_risk_of_poverty_or_social_exclusion

EUROSTAT (2015). Recent developments in unemployment at a European

and Member State level. Retrieved from

http://ec.europa.eu/eurostat/statistics-

explained/index.php/Unemployment_statistics

Fantuzzo, J., McWayne, C., \& Perry, M.A. (2004). Multiple dimensions of family involvement and their relations to behavioral and learning competencies for urban, low-income children. School Psychology Review, 33(4), 467-480. Retrieved from http://eportfoliocathymendoza.pbworks.com/f/Fantuzzo.pdf

Fine, M. (1993). [Ap]parent involvement: Reflections on parents, power and urban public schools. Teachers College Record, 94, 682-710.

Retrieved from http://www.tcrecord.org/Content.asp?ContentId=147

Flecha, R. (2000). Sharing Words. Theory and Practice of Dialogic Learning. Lanham: Rowman \& Littlefield.

Flecha, R. (2006-2011). INCLUD-ED. Strategies for inclusion and social cohesion from education in Europe. INTEGRATED PROJECT Priority 7 of Sixth Framework Programme. 
Flecha, R., \& Soler, M. (2013). Turning difficulties into possibilities: Engaging Roma families and students in school through dialogic learning. Cambridge Journal of Education, 3(44), 451-465. doi: 10.1080/0305764X.2013.819068

Flecha, R., \& Soler, M. (2014). Communicative Methodology: Successful Actions and Dialogic Politics. Current Sociology, 62(2), 232-242. doi: 10.1177/0011392113515141

Flecha, R. (2014-2018). IMPACT-EV. Evaluating the impact and outcomes of EUSSH research. 7h Framework Programme.

Fundación FOESSA (2014). VII Informe sobre exclusión y desarrollo social en España. Retrieved from http://www.foessa2014.es/informe/

García, C., Lastikka, A.L., \& Petreñas, C. (2013). Comunidades de aprendizaje. Scripta Nova-Revista Electrónica De Geografía $Y$ Ciencias Sociales, XVII (427). Retrieved from http://www.ub.edu/geocrit/sn/sn-427/sn-427-7.htm

Gatt, S., Ojala, M., \& Soler, M. (2011). Promoting social inclusion counting with everyone: Learning communities and INCLUD-ED. International Studies in Sociology of Education, 21(1), 33-47. doi: 10.1080/09620214.2011.543851

Gereluk, D., \& Race, R. (2007). Multicultural tensions in England, France and Canada: contrasting approaches and consequences. International Studies in Sociology of Education, 17(1-2), 113-129. doi:

10.1080/09620210701433837

Giroux, H., \& Flecha, R. (1992). Igualdad Educativa y Diferencia Cultural. Barcelona: El Roure.

Gómez, A., Puigvert, L., \& Flecha R. (2011). Critical Communicative Methodology: Informing real social transformation through research. Qualitative Inquiry, 17(3), 235-245. doi: 10.1177/1077800410397802

Green, M., \& Hulme, D. (2005). From correlates and characteristics to causes: Thinking about poverty from a chronic poverty perspective. 
112 Girbés-Peco et al. - De Escuela Gueto a Comunidad de Aprendizaje

World Development, 33(6), 867-879. doi:

10.1016/j.worlddev.2004.09.013

Habermas, J. (1984). Teoría de la acción comunicativa. Vol. 1:

Racionalidad de la acción y racionalización social. Vol. II: Crítica de la razón funcionalista. Madrid: Taurus.

Hammond, L. (2001). Notes from California: An anthropological approach to urban science education for language minority families. Journal of Research in Science Teaching, 38(9), 983-999. doi: 10.1002/tea.1043 Henderson, A. T., \& Mapp, K. L. (2002). A new wave of evidence: The impact of school, family, and community connections on student achievement. Austin, TX: National Center for Family \& Community Connections with School, Southwest.

Hidalgo, N. M., Eptein, J. L., \& Siu S. (2002). Research on families, schools and communities. A multicultural perspective. In J. A. Banks \& C. A. M. Banks (Eds.), Handbook for research on multicultural education (2nd ed., pp.631-55). San Franciso: Jossey-Bass.

Huat See, B., \& Gorard, S. (2013). What do rigorous evaluations tell us about the most promising parental involvement interventions? A critical review of what works for disadvantaged children in different age groups. England: Nuffi eld Foundation.

INCLUD-ED Consortium (2009). Actions for success in schools in Europe. Brussels: European Commission.

INCLUD-ED Consortium (2011). Actuaciones de éxito en las escuelas europeas. Madrid: IFIIE. Estudios CREADE.

Instituto Nacional de Estadística, INE (2012). Encuesta de condiciones de vida. Año 2012. datos provisionales. Retrieved from: http://www.ine.es/prensa/np740.pdf

Instituto Nacional de Estadística, INE (2013). Padrón de Municipal de Habitantes. Madrid: INE.

Instituto Nacional de Estadística, INE (2015). Encuesta de población activa$4^{\circ}$ trimestre 2014. Retrieved from http://www.ine.es/daco/daco42/daco4211/epa0414.pdf 
Izumi, L. T. (2002). They Have Overcome: High-Poverty, High-Performing Schools in California. San Francisco: Pacific Research Institute.

Jeynes, W. H. (2012). A meta-analysis of the efficacy of different types of parental involvement programs for urban students. Urban Education, 47(4), 706-742. doi: 10.1177/0042085912445643

Jencks, C., Bartlett, S., Corcoran, M., Crouse, J., Eaglesfield, D., Jackson, G., \& Williams, J. (1979). Who gets ahead?: The determinants of economic success in America. New York: Basic Books, Inc., Publishers.

Klugman, J., Rodríguez, F., \& Choi, H. (2011). The HDI 2010: New controversies, old critiques. The Journal of Economic Inequality, 9(2), 249-288. doi: 10.1007/s10888-011-9178-z

Mahoney, J., Lord, H., \& Carryl, E. (2005). An ecological analysis of afterschool program participation and the development of academic performance and motivational attributes for disadvantaged children. Child Development, 76(4), 811-25. doi: 10.1111/j.14678624.2005.00879.x

Maulucci, M. S. R. (2010). Resisting the marginalization of science in an urban school: Coactivating social, cultural, material, and strategic resources. Journal of Research in Science Teaching, 47(7), 840-860. doi: 10.1002/tea.20381

Nolan, B., \& Whelan, C. T. (2010). Using non-monetary deprivation indicators to analyze poverty and social exclusion: Lessons from Europe? Journal of Policy Analysis and Management 29(2), 305-325. doi: 10.1002/pam.20493

Oliver, E., \& Gatt, S. (2010). De los actos comunicativos de poder a los actos comunicativos dialógicos en las aulas organizadas en grupos interactivos. Signos, 43(2), 279-294. Retrieved from http://www.scielo.cl/pdf/signos/v43s2/a02.pdf

Orfield, G., \& Eaton, S. (1996). Dismantling Desegregation. The quiet reversal of Brown v. Board of Education. United States: The New Press. 
114 Girbés-Peco et al. - De Escuela Gueto a Comunidad de Aprendizaje

Orfield, G., \& Lee, C. (2005). Why segregation matters: Poverty and educational inequality. The Civil Rights Project. Harvard University. Retrieved from http://escholarship.org/uc/item/4xr8z4wb\#page-1

Phillips, D., \& Lowenstein, A. (2011). Early care, education, and child development. Annual Review of Psychology, 62, 483-500. doi: 10.1146/annurev.psych.031809.130707

PNUD (2014). Informe sobre Desarrollo Humano 2014. Sostener el progreso humano: reducir vulnerabilidades y construir resiliencia. New York: United Nations.

Puigvert, L., Christou, M., \& Holdford, J. (2012). Critical Communicative Methodology: including vulnerables voices in research through dialogue. Cambridge Journal of Education, 42(4), 513-526. doi: 10.1177/1077800410397802

Ramis, M., \& Krastina, L. (2010). Cultural intelligence in the school. Revista De Psicodidáctica, 15(2), 239-252. doi:

10.1387/RevPsicodidact.818

Rios, O. (2013). Sociocultural Transformation \& Development. Good Practices or Successful Actions. Multidisciplinary Journal of Educational Research, 3(2), 172-199. doi: 10.4471/remie.2013.11

Rose, P., \& Dyer, C. (2008). Chronic Poverty and Education: A Review of Literature Background Paper. Manchester: Chronic Poverty Research Centre.

Rothstein, R. (2004). Class and Schools. Food and Agriculture Organization of the United Nations. Retrieved from http://agris.fao.org/agrissearch/search.do?recordID=US201300122901

Santa Cruz, I., Siles, G., \& Vrecer, N. (2011). Invest for the long term or attend to immediate needs? Schools and the employment of less educated youths and adults. European Journal of Education, 46(2), 197-208. doi: 10.1111/j.1465-3435.2011.01475.x

Sen, A. (2000). Development as freedom. New York: Anchor Books. Stein, A., Freel, K., Hanson, A., Pacchiano, D., \& Eiland-Williford, B. (2013). The educare Chicago research-program partnership and 
follow-up study: Using data on program graduates to enhance quality improvement efforts. Early Education and Development, 24(1), 19-41. doi: 10.1080/10409289.2013.739542

Taylor, C., Power, S., \& Rees, G. (2010). Out-of-school learning: The uneven distribution of school provision and local authority support. British Educational Research Journal, 36(6), 1017-1036. doi: 10.1080/01411920903342046

Tellado, I., \& Sava, S. (2010). The role of non-expert adult guidance in the dialogic construction of knowledge. Revista De Psicodidáctica, 15(2). doi: 163-176. doi: 10.1387/RevPsicodidact. 822

Universidad Politécnica de Madrid (2010). Análisis urbanístico de Barrios Vulnerables en España. Madrid: Ministerio Ministerio de Fomento.

Valls, R., \& Kyriakides, L. (2013). The power of interactive groups: How diversity of adults volunteering in classroom groups can promote inclusion and success for children of vulnerable minority ethnic populations. Cambridge Journal of Education, 43(1), 17-33. doi: 10.1080/0305764X.2012.749213

Valls, R. (2012-2014). Actuaciones socioeducativas de éxito para la superación de la pobreza. Plan Nacional I+D+i. Secretaría de Estado de Universidades e Investigación. Ministerio de Educación y Ciencia.

Valls, R., \& Padrós, M. (2011). Using dialogic research to overcome poverty: From principles to action. European Journal of Education, 46(2), 173-183. doi: 10.1111/j.1465-3435.2011.01473.x

Wilson, W. J. (2003). Race, class and urban poverty: A rejoinder. Ethnic \& Racial Studies.26(6), 1096-1114. doi:

10.1080/0141987032000132522

Wilson, W. J. (2010). Why Both Social Structure and Culture Matter in a Holistic Analysis of Inner-City Poverty. The ANNALS of the American Academy of Political and Social Science, 629(1), 200-219. doi:10.1177/0002716209357403 
116 Girbés-Peco et al. - De Escuela Gueto a Comunidad de Aprendizaje

Wolbers, M. H. (2003). Job Mismatches and their Labour-Market Effects among School-Leavers in Europe. European Sociological Review, 19(3), 249-266. doi: 10.1093/esr/19.3.249

Sandra Girbés-Peco es profesora del Departamento de Didáctica y Organización Educativa de la Universidad de Barcelona

Fernando Macías-Aranda es profesor del Departamento de Teoría e Historia de la Educación de la Universidad de Barcelona

Pilar Álvarez-Cifuentes es profesora del Departamento de Teoría e Historia de la Educación de la Universidad de Barcelona

Dirección de contacto: Universidad de Barcelona. Facultad de Educación, Dpto Didáctica y Organización Educativa. Campus Mundet, Levante, Paseo Valle de Hebrón, 171, 2² dpcho 230 -08035 Barcelona. Email: sandra.girbes@ub.edu 